\title{
Behavioral Oscillations in Visual Attention Modulated by Task Difficulty
}

\author{
Airui Chen ${ }^{1,2+}$, Aijun Wang ${ }^{1,2+}$, Tianqi Wang ${ }^{1,2}$, Xiaoyu Tang ${ }^{3,4 *}$ and Ming Zhang ${ }^{1,2 *}$ \\ ${ }^{1}$ Research Center for Psychology and Behavioral Sciences, Soochow University, Suzhou, China, ${ }^{2}$ Department of \\ Psychology, Soochow University, Suzhou, China, ${ }^{3}$ School of Psychology, Liaoning Normal University, Dalian, China, \\ ${ }^{4}$ Liaoning Collaborative Innovation Center of Children and Adolescents Healthy Personality Assessment and Cultivation, \\ Dalian, China
}

The spotlight of attention is full of discrete moments and operates periodically. Recently, it has been well-documented there were behavioral oscillations in visual attention, however, different periodicities were demonstrated. Task difficulty may be an important factor causing disagreement in attentional periodic patterns. The present study examined behavioral oscillations in visual attention during difficult and easy

OPEN ACCESS

Edited by:

Eddy J. Davelaar,

Birkbeck University of London,

United Kingdom

Reviewed by:

Bao Zhang

Guangzhou University, China

Mark A. Elliott,

NUI Galway, Ireland

*Correspondence:

Ming Zhang

psyzm@suda.edu.cn

Xiaoyu Tang

tangyu-2006@163.com

tThese authors have contributed equally to this work.

Specialty section:

This article was submitted to Cognitive Science,

a section of the journal

Frontiers in Psychology

Received: 27 April 2017 Accepted: 04 September 2017 Published: 26 September 2017

Citation:

Chen A, Wang A, Wang T, Tang X and Zhang M (2017) Behavioral Oscillations in Visual Attention Modulated by Task Difficulty.

Front. Psychol. 8:1630. doi: 10.3389/fpsyg.2017.01630 tasks. A modified high temporal resolution cue-target paradigm in which the cue-target stimulus onset asynchrony (SOAs) varied from 0.1 to $1.08 \mathrm{~s}$ in steps of $20 \mathrm{~ms}$ was used. The target was detected with the accuracy of $65 \%$ in the difficult condition and $75 \%$ in the easy condition. Oscillatory patterns were analyzed and observed in behavioral performance. A theta rhythm was visible in the difficult version. However, attention oscillation increased to a higher frequency in the easy version. Task difficulty was negatively related to power for all bands. Our findings suggest that the attention spotlight switched faster when the task was easy, while, it switched much more slowly when the task was difficult in order to obtain more information. A flexible mechanism for attention spotlight was demonstrated, and task demand modulated attention oscillations.

Keywords: visual attention, attention spotlight, psychophysics, behavioral oscillations, task difficulty, flexible mechanism

\section{INTRODUCTION}

Visual system is confronted with large amounts of information in the environment. Despite this, we can live an effortless and well-ordered life. This ability is attributed to attention. Attention aids us to selectively focus on spatial locations, objects or features, prioritizing relevant information for enhanced processing while ignoring others, just like a spotlight (Posner, 1980), a Gaussian gradient (Downing and Pinker, 1985), or a zoom lens (Eriksen et al., 1990). However, the temporal structures of behavioral and neural representations of perception and attention have been ignored. Recent studies have strongly demonstrated that the spotlight of attention is full of discrete moments and operates periodically, regardless of whether attention focuses on a single location or multiple locations, which is called rhythmic sampling of visual attention (VanRullen et al., 2007; Busch and VanRullen, 2010; Landau and Fries, 2012; Fiebelkorn et al., 2013; Song et al., 2014; Landau et al., 2015; Dugué et al., 2015b, 2016; VanRullen, 2016b). The precise periodic structures were also revealed in a dynamic priming effect, which may be the cognitive basis of time perception (Elliott, 2014). 
One of the most important issues of rhythmic attention is the frequency of sampling. However, this issue remains poorly understood, and the rhythm was different across studies. Behavioral oscillations in participants' accuracy (ACC) suggested that attention periodicity was $8 \mathrm{~Hz}$ in a target detection task (Landau and Fries, 2012; Fiebelkorn et al., 2013). However, other researchers analyzed behavioral oscillations in participants' reaction time (RT) and observed a much higher frequency $(8-20 \mathrm{~Hz})$ in a target discrimination task (Song et al., 2014). A previous electroencephalography (EEG) study found that the phase of $7 \mathrm{~Hz}$ EEG activity just before flash onset modulated detection of the threshold flash under attention condition (Busch and VanRullen, 2010). The wagon wheel illusion was based on attentional motion computation at approximately $13 \mathrm{~Hz}$ (VanRullen et al., 2005). These attentional cycles also exist during visual searches in monkeys and humans (Buschman and Miller, 2009; Dugué et al., 2015a,b). A macaque monkey study found that attention shifts correlated with $18-34 \mathrm{~Hz}$ neural oscillations in the frontal eye fields (Buschman and Miller, 2009). Adopting psychophysics and transcranial magnetic stimulation (TMS) methods in human subjects revealed that attention periodically processed multiple search locations at approximately $5-10 \mathrm{~Hz}$ (Dugué et al., 2015a,b).

Task difficulty may be an important factor causing disagreement in attentional periodicities. A difficult 50\% threshold task was set up in some studies and $8 \mathrm{~Hz}$ rhythm was revealed in the time course of attention (Landau and Fries, 2012; Fiebelkorn et al., 2013). However, an easily completed task was used, thus the subjects' average accuracy was 98\%; an 8-20 Hz rhythm was found (Song et al., 2014). Two monkeys were well-trained in the easy search task, and their attention shifted at $18-34 \mathrm{~Hz}$ (Buschman and Miller, 2009). However, human subjects completed dual tasks, including a challenging search task and a probe detection task, and their attention worked at lower frequency bands (Dugué et al., 2015a,b). Task difficulty may modulate the amount of attention to the same stimuli, and it is measured and reflected in behavioral tasks and neuronal activity. Subjects' detectability was enhanced during difficult tasks (Urbach and Spitzer, 1995), and the response strength of inferior temporal and V4 neurons increased (Spitzer and Richmond, 1991; Boudreau et al., 2006).

The present study investigated whether task difficulty modulated attentional sampling behavior. We used a high temporal resolution cue-target paradigm to examine the temporal course of spatial attention. And, we used a threshold discrimination task rather than detection task because of decision biases in subjective criteria. Figure 1 shows that subjects covertly attended to two gratings to discriminate the targets' location in a two-alternative forced choice task (2AFC). Target discrimination performance in the difficult version was maintained at a $65 \%$ threshold by running a staircase procedure prior to the experiment. Target discrimination performance in the easy version was adjusted to a $75 \%$ threshold using the staircase method. Cue-to-target SOA ranged from 0.1 to $1.08 \mathrm{~s}$, in steps of $20 \mathrm{~ms}$, to achieve the temporal course of discrimination performance. The attention spotlight may switch much more slowly to obtain more information when the task is not easy and people have more difficulty in perceiving the stimulus if attention samples information from cluttered sensory environments in an intelligent and flexible manner. Thus, we predicted that manipulation of task difficulty would change the frequency of attention oscillations.

\section{MATERIALS AND METHODS}

Experiments were performed in accordance with the Declaration of Helsinki and approved by the ethical committee of Soochow University.

\section{Participants}

Thirty-one subjects (26 females, age 18-26 years, all righthanded) were recruited to participate in the study. Sixteen subjects participated in the difficult task, and 15 subjects performed the easy task. All subjects had normal or correctedto-normal vision. Participants gave informed consent prior to experiments in accordance with the Declaration of Helsinki and received monetary compensation afterward.

\section{Apparatus}

Subjects sat $70 \mathrm{~cm}$ from a 22-inch ViewSonic P225f CRT monitor $(1024$ pixel $\times 768$ pixel resolution at $100 \mathrm{~Hz})$ in a dark room with their heads stabilized in a chin rest. The experiment was programmed using the Psychophysics Toolbox (Brainard, 1997; Pelli, 1997) in Matlab. Responses were recorded through a keyboard.

\section{Stimuli}

The experimental scenario and parameters were adopted from Landau and Fries (2012). All stimuli were presented on a gray background $\left(3.88 \mathrm{~cd} / \mathrm{m}^{2}\right)$. Subjects were requested to maintain fixation at a white bull eye $\left(0.5^{\circ}\right.$ in diameter $)$ and covertly monitor two gratings ( $4^{\circ}$ in diameter) presented $5^{\circ}$ eccentrically on either side of the fixation to identify the target's location. The gratings, with spatial frequency $1.4 \mathrm{c} / \mathrm{deg}$ and contrast of $100 \%$, drifted at $0.7 \mathrm{c} / \mathrm{s}$. Drifting direction was randomized across trials (for reasons, see Landau and Fries, 2012, supplementary experimental procedures). The cue event, which consisted of four white disks (disk diameter: $1^{\circ} ; 1.5^{\circ}$ away from the grating's edge), was presented around either of the gratings for $30 \mathrm{~ms}$. The target event was a contrast decrement of 30-ms duration, which randomly occurred on the left or right grating. The amplitude of contrast decrement was determined using a QUEST staircase procedure for each subject.

\section{Experimental Procedure}

Fixation and gratings were presented at all times in each trial until red guess instruction onset. Cue events occurred randomly between 1 and $1.2 \mathrm{~s}$ from fixation and gratings onset. The target could appear at one of the 50 temporal intervals, in steps of $20 \mathrm{~ms}$, from 0.1 to $1.08 \mathrm{~s}$ after cue onset (SOA) to achieve a continuous monitoring assay of subjects' behavioral performance. The target randomly occurred on the cued side or the uncued side, with an equal likelihood of appearance. Subjects were informed of 50\% 


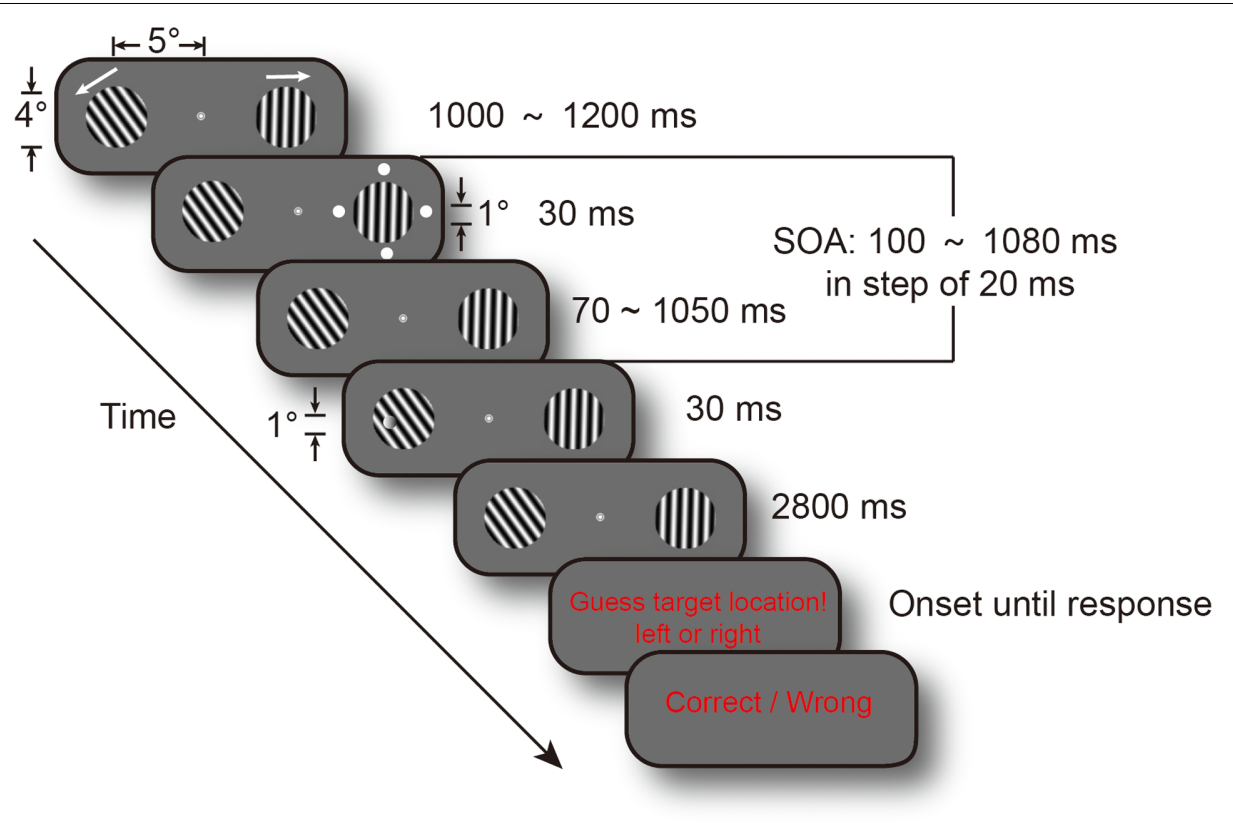

FIGURE 1 | Experimental procedure. Each trial display contained two drifting gratings, and their drifting directions were randomized across trials. Cue events, consisting of four white dots, occurred at a random time between 1 and $1.2 \mathrm{~s}$ from fixation and gratings onset. Target could appear at one of the 50 temporal intervals, in steps of $20 \mathrm{~ms}$, from 0.1 to $1.08 \mathrm{~s}$ after cue onset (SOA). Subjects were requested to determine target location, and their responses were recorded. The two arrows indicated gratings' drifting directions.

cue validity prior to the experiment. Performances were evaluated in a 2AFC task. Subjects were instructed to judge the location of the target (left arrow for target on the left grating; right arrow for right grating) on the keyboard during a period of $2.8 \mathrm{~s}$. Subjects who did not see the target guessed the location of the target and pressed either key. Visual feedback (correct or incorrect) was given after the subjects' response. Each subject completed 1600 trials in total, in eight blocks on two or three separate days to avoid a fatigue effect.

\section{Staircase Procedure}

The amplitude of target contrast decrement was determined using the QUEST procedure (Watson and Pelli, 1983), which determined the individual thresholds at which 65\% (difficult task) or $75 \%$ (easy task) of the stimuli were detected. The staircase procedure was identical to the experimental procedure except that no cue stimuli were presented. Each block had 60 trials, and each experimental condition (65 or $75 \%$ ) was measured three or four times.

\section{Data Analysis}

Behavioral data were analyzed using Matlab and the CircStat toolbox (Berens, 2009). Performance accuracy was calculated as a function of SOA to represent temporal profiles for each condition (cued vs. uncued). Response time greater than $5 \mathrm{~s}$ was excluded from analysis. Spectral analysis was performed on temporal profiles from 0.1 to $1.08 \mathrm{~s}$ after cue onset to examine the spatiotemporal dynamics of the time course. We adopted the analysis method which Song and his colleagues used in their study to detrend the ACC time course for each condition (Song et al., 2014). Slow trends were obtained by calculating $200 \mathrm{~ms}$ moving of average-ACC temporal profile, and then we removed these trend signals from the $980 \mathrm{~ms}$ epoch. This epoch was Hanning tapered, padded with zeros and Fourier transformed. The phase relation between cued and uncued conditions was calculated. Phase angle between cued and uncued conditions for a single subject was analyzed as a function of frequency from 0 to $20 \mathrm{~Hz}$. Betweensubject coherence in phase angles was calculated and Rayleigh tests were executed for phase differences in the $4 \sim 5 \mathrm{~Hz}$ band.

We used a non-parametric approach to assess the statistical significance of peaks in the spectra amplitude. Shuffling temporal profiles (ACC-SOA) separately within each subject 1000 times was performed to generate a randomization distribution. Surrogate signals were analyzed after each randomization in the same manner as the observed data. A permutation distribution for each frequency and significance thresholds $(p<0.05)$ were obtained.

\section{RESULTS}

Data in the difficult task were not normally distributed $[W(15)=0.821, p=0.007]$. Therefore, non-parametric statistical methods were used in the study to overcome the influence of outliers.

\section{Between-Subjects Analysis}

Sixteen subjects performed a difficult task (65\% version), and their average accuracy was $72.461 \%(S D=10.012 \%)$. Fifteen 

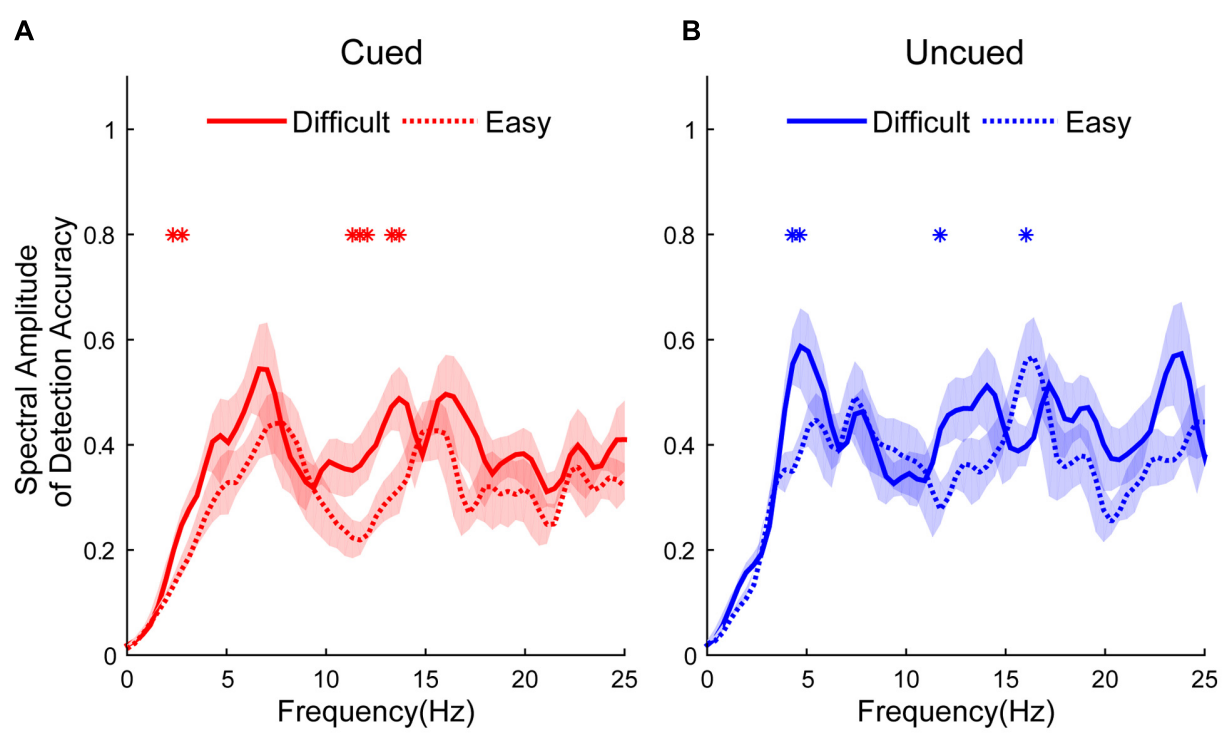

FIGURE 2 | Spectral amplitudes of detection accuracy from difficult and easy tasks (difficult: solid line; easy: dashed line) between cued and uncued conditions (cued condition: red line, shown in A; uncued condition: blue line, shown in B). Red and blue asterisks indicate the significant peak frequencies between spectral amplitudes in the easy and difficult tasks.

subjects participated in an easy task (75\% version), and their mean accuracy was $82.500 \%(S D=6.996 \%)$. A Mann-Whitney $U$-test indicated that ACC was higher in the easy task, with a large effect size $\left(U=39.000 ; N_{1}=16 ; N_{2}=15 ; p=0.001, r=0.5751\right)$.

Figure 2 shows the different spectral amplitudes between easy and difficult conditions. The amplitudes of the easy task in the cued condition were significantly lower than in the difficult task at 2.3438, 2.7344, 11.3281, 11.7188, 12.1094, 13.2813, and $13.6719 \mathrm{~Hz}$ (independent $t$-test, $p<0.05$, see Figure 2A). The amplitudes of the easy task in the uncued condition exhibited significantly lower amplitudes than the difficult task at 4.2969, 4.6875, and $11.7188 \mathrm{~Hz}$ but larger amplitudes at $16.0156 \mathrm{~Hz}$ (independent $t$-test, $p<0.05$, see Figure 2B). No differences were found at other frequencies.

Behavior performance was functionally related to the average amplitude of the delta-band $(1 \sim 4 \mathrm{~Hz})$, theta-band $(4 \sim 8 \mathrm{~Hz})$, alpha-band $(8 \sim 12 \mathrm{~Hz})$ and beta-band $(12 \sim 25 \mathrm{~Hz})$, as indicated by negative correlations (delta-band, $r=-0.5095, p=0.0034$; theta-band, $r=-0.5103, p=0.0034$; alpha-band, $r=-0.5621$, $p<0.001$, beta-band, $r=-0.8318, p<0.001$; see Figure 3).

\section{Within-Subject Analysis Difficult Task}

Figure 4 shows the raw ACC temporal courses as a function of cue-to-target SOAs under cued (red) and uncued (blue) conditions averaged across all subjects. Figures 4A,D show that a switching relationship between cued and uncued conditions was observed in the left visual field (LVF) and the right visual field (RVF). This result means that these two locations were being sampled alternatively, with better discrimination performance in one location and worse discrimination performance in the other location. Spectrotemporal analysis was performed separately in the left and right visual field to clarify temporal dynamics. Discrimination in the cued condition fluctuated at $6.6 \mathrm{~Hz}$ in the LVF (Figure 4B, red line; $p<0.05$ for $6.6406 \mathrm{~Hz}$ ) when the target occurred in the cued location. Discrimination in the uncued condition fluctuated at 4 and $19 \mathrm{~Hz}$ in the LVF (Figure 4B, blue line; $p<0.05$ for 4.29688-5.07813 and $18.75-19.1406 \mathrm{~Hz}$ ). A direct comparison between the peak frequencies for cued vs. uncued locations revealed no significant difference $[t(15)=1.357, p=0.195]$. The phase clustered around a mean of $85.3162 \pm 74.7776^{\circ}$ (Figure 4C). This phase was not significantly different from either 0 or $180^{\circ}$. Discrimination in the cued condition fluctuated at $7 \mathrm{~Hz}$ in the RVF (Figure $4 \mathrm{E}$, red line; $p<0.05$ for $7.0313 \mathrm{~Hz}$ ) when the target occurred in the cued location. Discrimination in the uncued condition fluctuated at 0.5 and $1 \mathrm{~Hz}$ in the RVF (Figure 4E, blue line; $p<0.05$ for $0.39063-0.78125$ and $1.1719 \mathrm{~Hz}$ ). A direct comparison between the peak frequencies for cued vs. uncued location revealed no significant difference $[t(15)=1.235, p=0.36]$. The phase of $4-5 \mathrm{~Hz}$ was clustered around a mean of $303.8575 \pm 63.6608^{\circ}$ (Figure 4F).

\section{Easy Task}

Figure 5 shows the raw ACC temporal courses as a function of cue-to-target SOAs under cued (red) and uncued (blue) conditions averaged across all subjects. No significant switching relationship patterns between cued and uncued conditions were observed. Discrimination in the cued condition fluctuated at 19.5 Hz when the cue occurred in the LVF (Figure 5B, red line; $p<0.05$ for $19.5313 \mathrm{~Hz}$ ) and the target occurred in the cued location. No significant frequencies were found in the uncued condition (Figure 5B, blue line). A direct comparison between the peak frequencies for cued vs. uncued locations revealed no significant differences $[t(14)=0.052, p=0.959]$. These 
cycles exhibited a phase relationship that clustered around a mean of $216.8592 \pm 65.7974^{\circ}$ (Figure 5C). This result was not significantly different from either 0 or $180^{\circ}$. Discrimination in the uncued condition fluctuated at $16.4 \mathrm{~Hz}$ in the RVF (Figure 5E, blue line; $p<0.05$ for $16.0156-16.7969 \mathrm{~Hz}$ ). No significant fluctuation was observed in the cued condition. A direct comparison between the peak frequencies for cued vs. uncued locations revealed that the fluctuation of the uncued condition was stronger than the cued condition $[t(14)=2.571, p=0.0226]$. These cycles exhibited a phase relationship that clustered around a mean of $9.0483 \pm 60.8117^{\circ}$ (Figure 5F).

\section{DISCUSSION}

The present study used a high temporal resolution cue-target paradigm to examine the temporal dynamics of behavioral time courses in visual attention during difficult and easy tasks. We demonstrated that behavior performance alternated at cued and uncued locations at a theta rhythm in the difficult task version. Our findings present evidence of a covert attentional sampling process. We found a theta rhythm in the difficult task version, which is consistent with previous studies (Landau and Fries, 2012; Fiebelkorn et al., 2013) and suggests covert attention samples multiple locations periodically. The attention period increased to a high frequency in the easy task version. The results of between-subjects analysis demonstrated that task difficulty was related to power for the delta-band $(1 \sim 4 \mathrm{~Hz})$, thetaband $(4 \sim 8 \mathrm{~Hz})$, alpha-band $(8 \sim 12 \mathrm{~Hz})$, and beta-band $(12 \sim 25 \mathrm{~Hz})$. Specifically, subjects with better performance exhibited less power. The results of within-subjects and betweensubjects analysis consistently demonstrated that task difficulty influenced the pattern of attention oscillations. The attention spotlight may switch faster when the task is easy, and it may switch much slower when the task is difficult in order to obtain more information. These results suggest that attention spotlight
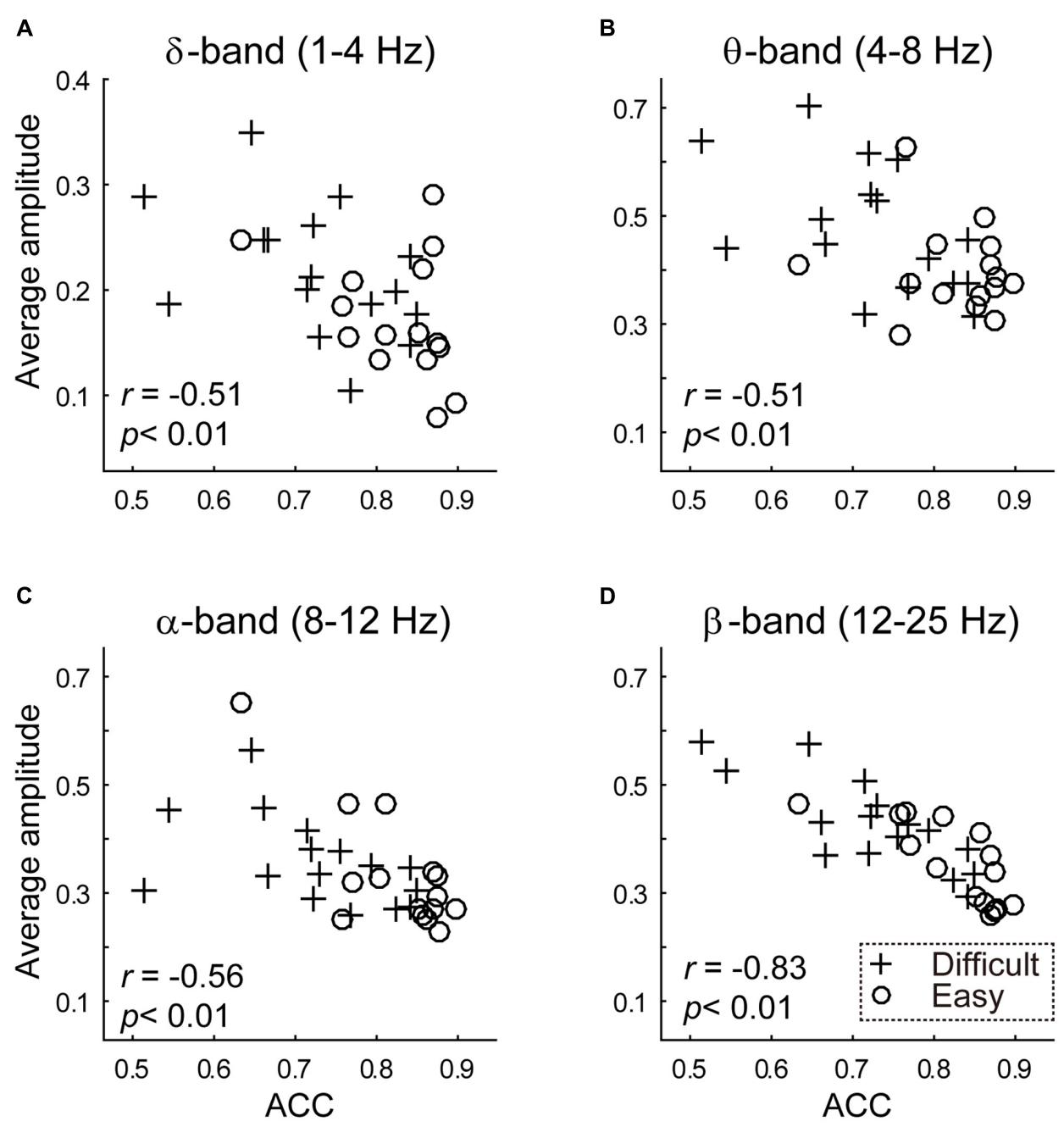

FIGURE 3 | The results of between-subject analysis are plotted. The scatterplot between transformed $\mathbf{( A )}$ delta band $(1 \sim 4 \mathrm{~Hz})(\mathbf{B})$ theta band $(4 \sim 8 \mathrm{~Hz})(\mathbf{C})$ alpha band $(8 \sim 12 \mathrm{~Hz})(\mathbf{D})$ beta band $(12 \sim 25 \mathrm{~Hz}$ ) average amplitude (y-axis) and discrimination accuracy ( $\mathrm{x}$-axis). Average amplitudes were calculated using a Fast Fourier Transformation. Crossed and circles illustrated average amplitudes in the difficult and easy task, respectively. 




samples information from cluttered sensory environments in an intelligent and flexible manner.

The time-quantum model (TQM) assumes the existence of an absolute lower bound for intermittencies, the timequantum, as an (approximately) universal constant that has a duration of approximately $4.5 \mathrm{~ms}$. Any admissible intermittencies are integer multiples of the quantum (Geissler, 1987). Elliott found that the priming effect achieved strongest when the dynamic priming frequencies synchronized with $6.69 \mathrm{~Hz}$ (149, approximately 33 times of $4.5 \mathrm{~ms}$ ) rhythm (Elliott, 2014). 

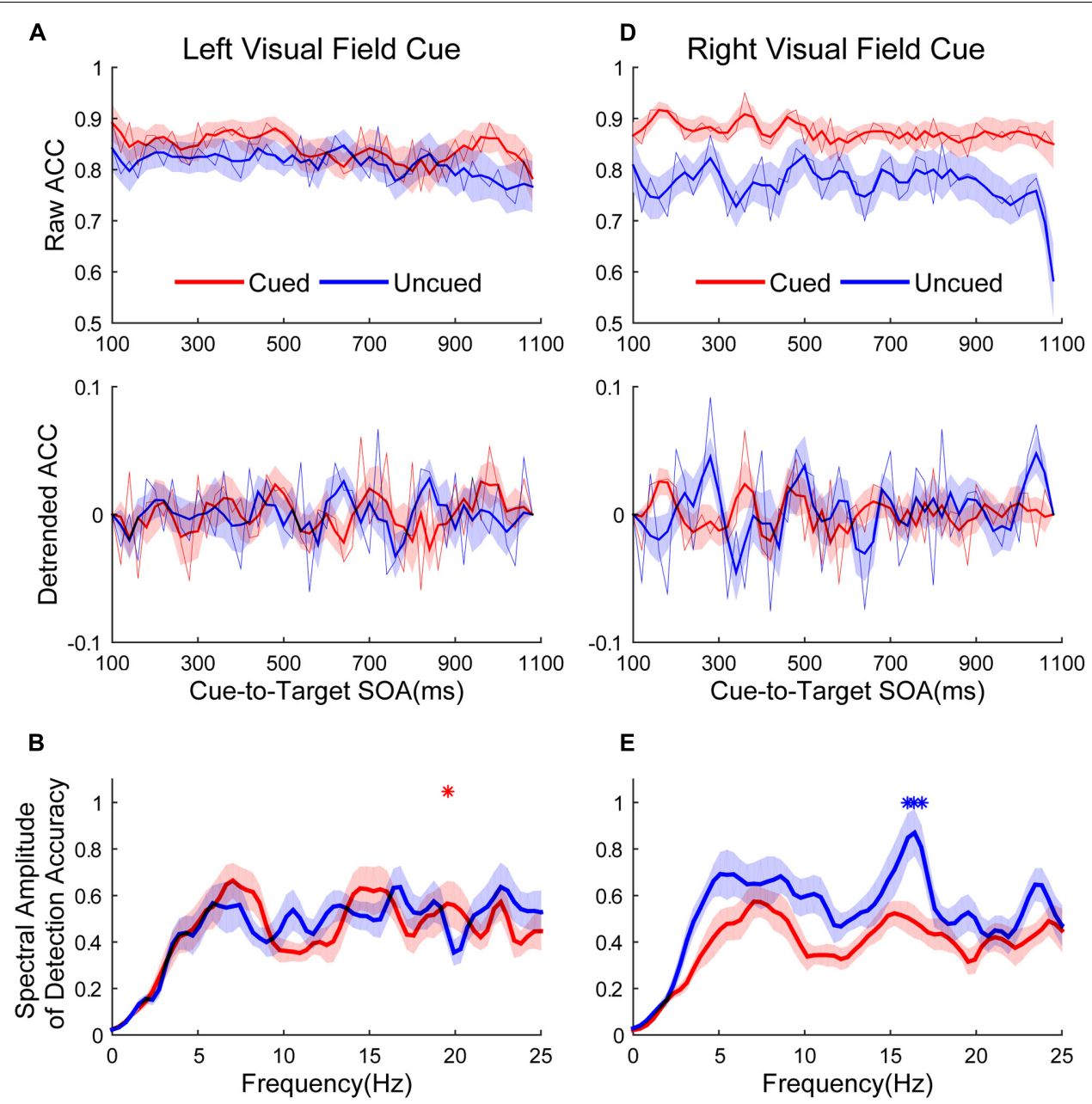

C

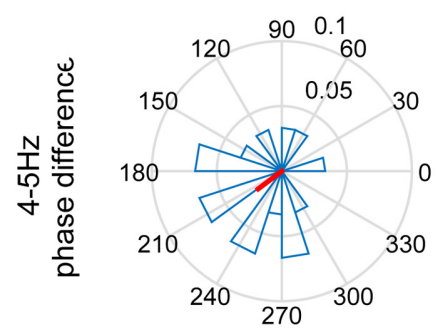

$\mathbf{F}$



C-UC phase mean $=216.8592$

Rayleigh test, $p=0.17684$

C-UC phase mean $=9.0483$

Rayleigh test, $p=0.054742$

FIGURE 5 | The results of ACC time course, spectral amplitude and phase relationships between cued and uncued in easy task. The left three figures represent data in the LVF. (A) The raw ACC time course and detrended ACC time course as a function of cue-to-target SOAs under cued condition (red) and uncued (blue) conditions averaged across all subjects. (B) A frequency domain representation of the behavioral data shown in (A). (C) Phase coherence between the cued and uncued conditions. Each subject's phase difference is plotted on the circle, with the average difference plotted. (D-F) Represent when the cue event occurred in the RVF, corresponding to (A-C).

This author thought that the conscious realization of events was mediated sometimes by anticipatory cognition and sometimes by cognition, which occurred subsequent to the coded event. Our study demonstrated that the different oscillation pattern between difficult and easy tasks, thus indicated that attention oscillations were modulated by task difficulty. Dugué and colleagues found that search type modulated processing in a visual search. Alpha frequency predominated for feature search, and a theta frequency was observed for a conjunction search (Dugué et al., 2017). These results further suggest that attention and perception rhythm are 
not constant and may be modulated by anticipatory cognition, task difficulty, and other cognitive factors.

Neural oscillations in several brain regions and networks play important roles in perception and cognition (Buzsáki, 2006; Arnal and Giraud, 2012; Fries, 2015). Rhythmic frame by oscillations may force the sensory system to use the nested temporal structure within each oscillatory cycle. Concentrating neuronal resources at specific moments in time while sparing these resources at other time points may be economic (VanRullen, 2016a). We may assume that task difficulty mediates the allocation of neural recourses to influence attention oscillations in behavior. This speculation requires testing in further experiments.

Notably, we used a 2AFC task instead of a detection task to better avoid subjects' criteria (conservative or risky strategy). Participants in the detection task reported "Did the target appear or not?" Subjects who adopted a riskier strategy would likely report that the target appeared, which created a high false alarm probability. Subjects who adopted a more conservative strategy tended to report that the target did not appear, which created low hit ratios (Drewes et al., 2015). Our improved methodology greatly avoided decision bias toward the oscillatory patterns. Previous studies used a detection task, and the present study used a $2 \mathrm{AFC}$ task. Both tasks found a $4-\mathrm{Hz}$ oscillatory pattern in the 50\% threshold version (Landau and Fries, 2012; Fiebelkorn et al., 2013). This result suggests our finding was the oscillating nature of attention rather than subjects' decision fluctuations. Future studies should use more elaborate methods to investigate the mechanisms of attention. In the study, saccades or microsaccades were not monitored in our experiments. However, the results are likely not due to these exogenous attention effects. Saccades are executed as part of ongoing attentional rhythm instead of an inducer (Hogendoorn, 2016). The function of saccades or microsaccades generally manifest on the low frequency and cannot explain the high frequency of the easy version in the study.

Our findings suggest a flexible mechanism for attention spotlight that may be modulated by task demand. We speculate that task demand is akin to the brake pedal (slowdown) or

\section{REFERENCES}

Arnal, L. H., and Giraud, A.-L. (2012). Cortical oscillations and sensory predictions. Trends Cogn. Sci. 16, 390-398. doi: 10.1016/j.tics.2012.05.003

Berens, P. (2009). CircStat: a MATLAB toolbox for circular statistics. J. Stat. Softw. 31, 1-21. doi: 10.18637/jss.v031.i10

Boudreau, C. E., Williford, T. H., and Maunsell, J. H. R. (2006). Effects of task difficulty and target likelihood in area V4 of macaque monkeys. J. Neurophysiol. 96, 2377-2387. doi: 10.1152/jn.01072.2005

Brainard, D. H. (1997). The psychophysics toolbox. Spat. Vis. 10, 433-436. doi: $10.1163 / 156856897 X 00357$

Busch, N. A., and VanRullen, R. (2010). Spontaneous EEG oscillations reveal periodic sampling of visual attention. Proc. Natl. Acad. Sci. U.S.A. 107, 16048-16053. doi: 10.1073/pnas.1004801107

Buschman, T. J., and Miller, E. K. (2009). Serial, covert shifts of attention during visual search are reflected by the frontal eye fields and correlated with population oscillations. Neuron 63, 386-396. doi: 10.1016/j.neuron.2009.06.020

Buzsáki, G. (2006). Rhythms of the Brain. New York, NY: Oxford University Press. doi: 10.1093/acprof:oso/9780195301069.001.0001 accelerator pedal (speedup) of the attention sampling machine. Further studies should investigate when and where the discrete attentional sampling occurs in our visual hierarchy.

\section{ETHICS STATEMENT}

The study was supported by Ethics Committee of Soochow University (ECSU). Firstly, the experimenter explained to participants and promised that the rights of individuals to privacy, confidentiality, and self-determination. Participants were informed that your participation in this research is entirely voluntary, and this experiment is totally harmless. Secondly, the experimenter explained what participants have to do in this research. Each participant gave written informed consent.

\section{AUTHOR CONTRIBUTIONS}

AC and MZ designed the research. AC and TW performed the research. AC analyzed the data. AC and AW wrote the manuscript text. MZ and XT reviewed the manuscript.

\section{FUNDING}

This research was supported by the National Natural Science Foundation of China (31371025 and 31600882). AW was supported by the National Natural Science Foundation of China (31700939), the China Postdoctoral Science Foundation (2017M611888), the Natural Science Foundation of Jiangsu Province (BK20170333) and MOE Project of Humanities and Social Sciences (17YJC190024).

\section{ACKNOWLEDGMENT}

The authors express gratitude to Bo Dong and Peijun Yuan for their help with data analysis.

Downing, C. J., and Pinker, S. (1985). “The spatial structure of visual attention," in Attention and Performance XI, eds M. I. Posner and O. S. M. Marin (Hillsdale, NJ: Erlbaum), 171-188.

Drewes, J., Zhu, W., Wutz, A., and Melcher, D. (2015). Dense sampling reveals behavioral oscillations in rapid visual categorization. Sci. Rep. 5:16290. doi: $10.1038 /$ srep 16290

Dugué, L., Marque, P., and VanRullen, R. (2015a). Theta oscillations modulate attentional search performance periodically. J. Cogn. Neurosci. 27, 945-958. doi: 10.1162/jocn_a_00755

Dugué, L., McLelland, D., Lajous, M., and VanRullen, R. (2015b). Attention searches nonuniformly in space and in time. Proc. Natl. Acad. Sci. U.S.A. 112, 15214-15219. doi: 10.1073/pnas.1511331112

Dugué, L., Roberts, M., and Carrasco, M. (2016). Attention reorients periodically. Curr. Biol. 26, 1595-1601. doi: 10.1016/j.cub.2016.04.046

Dugué, L., Xue, A. M., and Carrasco, M. (2017). Distinct perceptual rhythms for feature and conjunction searches. J. Vis. 17:22. doi: 10.1167/17.3.22

Elliott, M. A. (2014). Atemporal equilibria: pro- and retroactive coding in the dynamics of cognitive microstructures. Front. Psychol. 5:990. doi: 10.3389/fpsyg. 2014.00990 
Eriksen, C. W., Webb, J. M., and Fournier, L. R. (1990). How much processing do nonattended stimuli receive? Apparently very little, but... Percept. Psychophys. 47, 477-488. doi: 10.3758/BF03208181

Fiebelkorn, I. C., Saalmann, Y. B., and Kastner, S. (2013). Rhythmic sampling within and between objects despite sustained attention at a cued location. Curr. Biol. 23, 2553-2558. doi: 10.1016/j.cub.2013. 10.063

Fries, P. (2015). Rhythms for cognition: communication through coherence. Neuron 88, 220-235. doi: 10.1016/j.neuron.2015.09.034

Geissler, H.-G. (1987). The temporal architecture of central information processing: evidence for a tentative time-quantum model. Psychol. Res. 49, 99-106. doi: 10.1007/BF00308674

Hogendoorn, H. (2016). Voluntary saccadic eye movements ride the attentional rhythm. J. Cogn. Neurosci. 28, 1625-1635. doi: 10.1162/jocn

Landau, A. N., and Fries, P. (2012). Attention samples stimuli rhythmically. Curr. Biol. 22, 1000-1004. doi: 10.1016/j.cub.2012.03.054

Landau, A. N., Schreyer, M. H., van Pelt, S., and Fries, P. (2015). Distributed attention is implemented through theta-rhythmic gamma modulation. Curr. Biol. 25, 2332-2337. doi: 10.1016/j.cub.2015. 07.048

Pelli, D. G. (1997). The video toolbox software for visual psychophysics: transforming numbers into movies. Spat. Vis. 10, 437-442. doi: 10.1163/ 156856897 X00366

Posner, M. I. (1980). Orienting of attention. Q. J. Exp. Psychol. 32, 3-25. doi: 10.1080/00335558008248231

Song, K., Meng, M., Chen, L., Zhou, K., and Luo, H. (2014). Behavioral oscillations in attention: rhythmic $\alpha$ pulses mediated through $\theta$ band. J. Neurosci. 34, 4837-4844. doi: 10.1523/JNEUROSCI.4856-13.2014
Spitzer, H., and Richmond, B. J. (1991). Task difficulty: ignoring, attending to, and discriminating a visual stimulus yield progressively more activity in inferior temporal neurons. Exp. Brain Res. 83, 340-348. doi: 10.1007/BF00231157

Urbach, D., and Spitzer, H. (1995). Attentional effort modulated by task difficulty. Vision Res. 35, 2169-2177. doi: 10.1016/0042-6989(94)00305-X

VanRullen, R. (2016a). Perceptual cycles. Trends Cogn. Sci. 20, 723-735. doi: 10.1016/j.tics.2016.07.006

VanRullen, R. (2016b). "Perceptual rhythms," in Stevens Handbook of Experimental Psychology, ed. J. Serences (Hoboken, NJ: Wiley).

VanRullen, R., Carlson, T., and Cavanagh, P. (2007). The blinking spotlight of attention. Proc. Natl. Acad. Sci. U.S.A. 104, 19204-19209. doi: 10.1073/pnas. 0707316104

VanRullen, R., Reddy, L., and Koch, C. (2005). Attention-driven discrete sampling of motion perception. Proc. Natl. Acad. Sci. U.S.A. 102, 5291-5296. doi: 10.1073/ pnas.0409172102

Watson, A. B., and Pelli, D. G. (1983). Quest: a Bayesian adaptive psychometric method. Percept. Psychophys. 33, 113-120. doi: 10.3758/BF03202828

Conflict of Interest Statement: The authors declare that the research was conducted in the absence of any commercial or financial relationships that could be construed as a potential conflict of interest.

Copyright (C) 2017 Chen, Wang, Wang, Tang and Zhang. This is an open-access article distributed under the terms of the Creative Commons Attribution License (CC BY). The use, distribution or reproduction in other forums is permitted, provided the original author(s) or licensor are credited and that the original publication in this journal is cited, in accordance with accepted academic practice. No use, distribution or reproduction is permitted which does not comply with these terms. 